\title{
Constant terms of near-Dyson polynomials
}

\author{
Alexey Gordeev \\ Saint Petersburg Department of the Steklov Mathematical Institute \\ Saint Petersburg, Russia \\ gordalserg@gmail.com
}

Submitted: Jun 9, 2017; Accepted: Oct 6, 2018; Published: Oct 19, 2018

(c) The author. Released under the CC BY-ND license (International 4.0).

\begin{abstract}
We formulate and prove a formula for the constant term for a certain class of Laurent polynomials, which include the Dyson conjecture and its generalizations by Bressoud and Goulden. Our method is explicit Combinatorial Nullstellensatz.
\end{abstract}

\section{Mathematics Subject Classifications: 11B65}

We recall the following form of Alon's Combinatorial Nullstellensatz (appeared recently in $[13,14,15]$, but essentially going back to Jacobi [8], see [9] for a modern exposition). It proved to be very useful $[5,11,12,13]$ for finding coefficients of polynomials.

Theorem 1. Let $f\left(x_{1}, \ldots, x_{n}\right)$ be a polynomial over a field $\mathbb{F}$ of degree $\leqslant d_{1}+\cdots+d_{n}$.

Consider the grid $G=G_{1} \times \cdots \times G_{n}, G_{i} \subset \mathbb{F}, \# G_{i}=d_{i}+1$. The coefficient of $\prod_{i=1}^{n} x_{i}^{d_{i}}$ in $f$ is

$$
\sum_{\left(c_{1}, \ldots, c_{n}\right) \in G} \frac{f\left(c_{1}, \ldots, c_{n}\right)}{\prod_{i=1}^{n} \prod_{z \in G_{i} \backslash\left\{c_{i}\right\}}\left(c_{i}-z\right)} .
$$

Let $x_{1}, \ldots, x_{n}, q$ be commuting indeterminates. Consider a Laurent polynomial $f\left(x_{1}, \ldots, x_{n}, q\right)$ over a field $\mathbb{F}$. Denote its constant term over the function field $\mathbb{F}(q)$ as $C T[f]$.

Let $a_{1}, \ldots, a_{n}$ be non-negative integers, $a=a_{1}+\cdots+a_{n}$. In a seminal 1962 work [4] Dyson formulated the following conjecture:

$$
C T\left[\prod_{i \neq j}\left(1-x_{i} / x_{j}\right)^{a_{i}}\right]=\frac{a !}{\prod_{i=1}^{n} a_{i} !}
$$

This was proved by Gunson [unpublished] and Wilson [16] in the same year. The elegant proof, based on Lagrange interpolation, was given by Good [7]. In [13] another proof based on the above-stated form of Combinatorial Nullstellensatz is given. It was generalized to a $q$-version (proved for the first time in [17] by a different method) in [11]. 
Constant term identities with Laurent polynomials (such as this one) often arise in quantum electrodynamics. They are also closely related to Selberg-type integrals, which play an important role in random matrix theory, statistical mechanics and special function theory (see the exposition in [6]).

There are versions of (a particular case of) Dyson's conjecture for arbitrary root systems, in which Dyson's original case corresponds to $A_{n}$. These are famous Macdonald's conjectures proved by Cherednik [3] with the help of the so-called double affine Hecke algebras.

Understanding for which Laurent polynomials such identities do exist is an important question. The application of Combinatorial Nullstellensatz allowed to make substantial progress in this area, and our results continue this development.

We start with recalling the proof of $q$-version of the Dyson conjecture. Define $[l, r]=$ $\{l, l+1, \ldots, r\}$. Let $\chi(\ldots)$ be equal to 1 if the expression in the parentheses is true, and to 0 otherwise. Also, denote $(x)_{n}=\prod_{t=0}^{n-1}\left(1-q^{t} x\right)$.

Theorem 2. Let $a_{1}, \ldots, a_{n}$ be non-negative integers, $a=a_{1}+\cdots+a_{n}$. Consider the Laurent polynomial

$$
f\left(x_{1}, \ldots, x_{n}, q\right)=\prod_{1 \leqslant i<j \leqslant n}\left(x_{i} / x_{j}\right)_{a_{i}}\left(q x_{j} / x_{i}\right)_{a_{j}}
$$

over a field $\mathbb{F}$. Then

$$
C T[f]=\frac{(q)_{a}}{(q)_{a_{1}}(q)_{a_{2}} \cdots(q)_{a_{n}}} .
$$

Proof. We can assume that all $a_{i}>0$ (if $a_{i}=0$, then each factor of $f$ contains $x_{i}$ only in non-negative degree. Since we are interested in the constant term of $f$, we can assume that $f$ does not depend on $x_{i}$ ).

$C T[f]$ equals to the coefficient of $\prod_{i=1}^{n} x_{i}^{a-a_{i}}$ in the polynomial $h$, where

$$
h\left(x_{1}, \ldots, x_{n}, q\right)=\prod_{1 \leqslant i<j \leqslant n}\left(x_{i} / x_{j}\right)_{a_{i}}\left(q x_{j} / x_{i}\right)_{a_{j}} \times x_{j}^{a_{i}} x_{i}^{a_{j}} .
$$

We will calculate this coefficient of $h$ using Theorem 1 .

Consider the grid

$$
G=\left\{\left(q^{y_{1}}, \ldots, q^{y_{n}}\right) \mid y_{i} \in R_{i}\right\}
$$

where

$$
R_{i}=\left[0, a-a_{i}\right]
$$

Let us assume that $x=\left(x_{1}, \ldots, x_{n}\right)=\left(q^{y_{1}}, \ldots, q^{y_{n}}\right)=q^{y} \in G$ is not a zero of $h$. Then, for each $i<j$,

$$
y_{j}-y_{i} \geqslant a_{i} \text { or } y_{i}-y_{j} \geqslant a_{j}+1,
$$

otherwise one of the factors in $\left(x_{i} / x_{j}\right)_{a_{i}}\left(q x_{j} / x_{i}\right)_{a_{j}} \times x_{j}^{a_{i}} x_{i}^{a_{j}}$ equals to zero.

In particular, it means that all $y_{i}$ are pairwise distinct. Let $\pi \in S_{n}$ be such a permutation that

$$
y_{\pi_{1}}<y_{\pi_{2}}<\cdots<y_{\pi_{n}}
$$


We know that

$$
y_{\pi_{i+1}}-y_{\pi_{i}} \geqslant a_{\pi_{i}}+\chi\left(\pi_{i+1}<\pi_{i}\right) .
$$

Adding up these inequalities and taking into account that $y_{\pi_{1}} \geqslant 0$, we get

$$
y_{\pi_{n}}-y_{\pi_{1}} \geqslant a-a_{\pi_{n}}+\sum_{i=1}^{n-1} \chi\left(\pi_{i+1}<\pi_{i}\right) .
$$

But $y_{\pi_{n}} \leqslant a-a_{\pi_{n}}$, so $\pi_{i}<\pi_{i+1}$ for all $i$, which means that $\pi=i d$.

Let us note that all intermediate inequalities have to become equalities, so the only point on the grid which is not a zero of $h$ is $q^{y}$, where $y_{i}=a_{1}+a_{2}+\cdots+a_{i-1}$.

Define for convenience $y_{n+1}=a$. By Theorem 1 ,

$$
\begin{aligned}
C T[f] & =\left(\prod_{1 \leqslant i<j \leqslant n}\left(q^{y_{i}-y_{j}}\right)_{a_{i}}\left(q^{y_{j}+1-y_{i}}\right)_{a_{j}} \times q^{y_{j} a_{i}} q^{y_{i} a_{j}}\right) /\left(\prod_{i=1}^{n} \prod_{z \in\left[0, a-a_{i}\right] \backslash\left\{y_{i}\right\}}\left(q^{y_{i}}-q^{z}\right)\right) \\
& =\frac{\left(\prod_{1 \leqslant i<j \leqslant n}\left(\prod_{k=0}^{a_{i}-1}\left(q^{y_{j}}-q^{y_{i}+k}\right) \times \prod_{k=0}^{a_{j}-1}\left(q^{y_{i}}-q^{y_{j}+1+k}\right)\right)\right)}{\left(\prod_{i=1}^{n}(-1)^{y_{i}}\left(\prod_{t=0}^{y_{i}-1} q^{t}\right)(q)_{y_{i}} q^{y_{i}\left(a-a_{i}-y_{i}\right)}(q)_{a-a_{i}-y_{i}}\right)} \\
& =\frac{\left(\prod_{1 \leqslant i<j \leqslant n}\left((-1)^{a_{i}}\left(\prod_{t=y_{i}}^{y_{i+1}-1} q^{t}\right) \frac{(q)_{y_{j}-y_{i}}}{(q)_{y_{j}-y_{i+1}}} \times q^{y_{i} a_{j}} \frac{(q)_{y_{j+1}-y_{i}}}{(q)_{y_{j}-y_{i}}}\right)\right)}{\left(\prod_{i=1}^{n}(-1)^{y_{i}}\left(\prod_{t=0}^{y_{i}-1} q^{t}\right)(q)_{y_{i}-y_{1}} q^{y_{i}\left(a-a_{i}-y_{i}\right)}(q)_{y_{n+1}-y_{i+1}}\right)} \\
& =(q)_{y_{n+1}-y_{1}} /\left(\prod_{i=1}^{n}(q)_{y_{i+1}-y_{i}}\right)=\frac{(q)_{a}}{(q)_{a_{1}} \cdots(q)_{a_{n}}} .
\end{aligned}
$$

Next we give simple proofs of the master theorem and its transitive analogue from [2] using a similar technique. A similar proof in a different context can be found in [10] (see Theorems 1.2 and 3.5), though our proof is more direct.

A tournament $T$ on $n$ vertices is a set of ordered pairs $(i, j)$ such that $1 \leqslant i \neq j \leqslant n$ and $(i, j) \in T$ if and only if $(j, i) \notin T$. One way of interpreting a tournament is as a relation on a set $[1, n]: i \rightarrow j$ if and only if $(i, j) \in T$.

A tournament $T$ is transitive if the relation $\rightarrow$ is transitive. For a transitive tournament $T$, a winner permutation $\sigma \in S_{n}$ is such a permutation that $\sigma_{i} \rightarrow \sigma_{j}$ if and only if $i<j$. Note that every transitive tournament $T$ has a unique winner permutation $\sigma=\sigma(T)$.

Theorem 3. Let $T$ be a tournament on $n$ vertices. Let $a_{1}, \ldots, a_{n}$ be positive integers, $a=a_{1}+\cdots+a_{n}$. Consider the Laurent polynomial

$$
f\left(x_{1}, \ldots, x_{n}, q\right)=\prod_{(i, j) \in T}\left(x_{i} / x_{j}\right)_{a_{i}}\left(q x_{j} / x_{i}\right)_{a_{j}-1}
$$

over a field $\mathbb{F}$. If $T$ is nontransitive, then $C T[f]=0$. If $T$ is transitive with a winner permutation $\sigma$, then

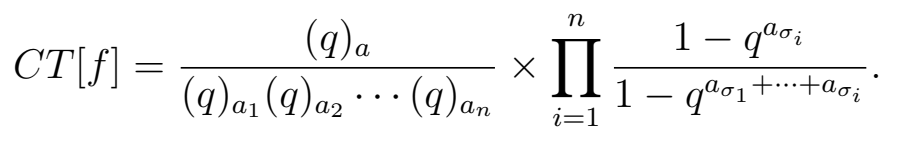


Proof. Let $\operatorname{deg}(i)=\#\{j \mid(i, j) \in T\}$. Consider a permutation $\delta \in S_{n}$ such that for each $1 \leqslant i<j \leqslant n \operatorname{deg}\left(\delta_{i}\right) \geqslant \operatorname{deg}\left(\delta_{j}\right)$, and $\operatorname{deg}\left(\delta_{i}\right)=\operatorname{deg}\left(\delta_{j}\right)$ only when $\delta_{i}<\delta_{j}$. Note that $\sigma=\delta$ in the case when $T$ is transitive.

$C T[f]$ equals to the coefficient of $\prod_{i=1}^{n} x_{i}^{a-a_{i}-\operatorname{deg}(i)}$ in the polynomial $h$, where

$$
h\left(x_{1}, \ldots, x_{n}, q\right)=\prod_{(i, j) \in T}\left(x_{i} / x_{j}\right)_{a_{i}}\left(q x_{j} / x_{i}\right)_{a_{j}-1} \times x_{j}^{a_{i}} x_{i}^{a_{j}-1} .
$$

Once again, we will calculate this coefficient using Theorem 1.

Consider the grid

$$
G=\left\{\left(q^{y_{1}}, \ldots, q^{y_{n}}\right) \mid y_{i} \in R_{i}\right\}
$$

where

$$
\begin{gathered}
R_{i}=\left[0, a-a_{i}\right] \backslash S_{i}, \\
S_{\delta_{i}}=\left\{a-a_{\delta_{i}}-\sum_{v=j}^{n} a_{\delta_{v}} \mid n+1-\operatorname{deg}\left(\delta_{i}\right)<j \leqslant n+1\right\} .
\end{gathered}
$$

Assume that $x=\left(x_{1}, \ldots, x_{n}\right)=\left(q^{y_{1}}, \ldots, q^{y_{n}}\right)=q^{y} \in G$ is not a zero of $h$. For each $(i, j) \in T$,

$$
y_{j}-y_{i} \geqslant a_{i} \text { or } y_{i}-y_{j} \geqslant a_{j}
$$

otherwise one of the factors in $\left(x_{i} / x_{j}\right)_{a_{i}}\left(q x_{j} / x_{i}\right)_{a_{j}-1} \times x_{j}^{a_{i}} x_{i}^{a_{j}-1}$ equals to zero.

It follows that all $y_{i}$ are pairwise distinct. Let $\pi \in S_{n}$ be such a permutation that

$$
y_{\pi_{1}}<y_{\pi_{2}}<\cdots<y_{\pi_{n}}
$$

We know that

$$
y_{\pi_{i+1}}-y_{\pi_{i}} \geqslant a_{\pi_{i}} .
$$

Adding up these inequalities and taking into account that $y_{\pi_{1}} \geqslant 0$, we get

$$
y_{\pi_{n}}-y_{\pi_{1}} \geqslant a-a_{\pi_{n}}
$$

But $y_{\pi_{n}} \leqslant a-a_{\pi_{n}}$, so all intermediate inequalities have to become equalities, and

$$
y_{\pi_{i}}=a-\sum_{j=i}^{n} a_{\pi_{j}} .
$$

Since $y_{\pi_{n}} \notin S_{\pi_{n}}$, from definition of $S_{\pi_{n}}$ it follows that $\operatorname{deg}\left(\pi_{n}\right)=0$. But $T$ is a tournament, so $\operatorname{deg}(i)=0$ for at most one $i$. Since $q^{y}$ is not a zero of $h$, such $i$ exists (and equals to $\left.\delta_{n}\right)$, so $\operatorname{deg}\left(\delta_{n}\right)=0$ and $\pi_{n}=\delta_{n}$.

Assume that we have already showed that $\pi_{k}=\delta_{k}$ and $\operatorname{deg}\left(\delta_{k}\right)=n-k$ for $j<k \leqslant n$. Note that these conditions on deg imply that $\left(\delta_{i}, \delta_{k}\right) \in T$ for all $1 \leqslant i<k, j<k \leqslant n$. Then $\operatorname{deg}\left(\delta_{i}\right) \geqslant n-j$ for all $1 \leqslant i \leqslant j$, and, since $T$ is a tournament, $\operatorname{deg}\left(\delta_{i}\right)>n-j$ for all $1 \leqslant i<j$. 
$y_{\pi_{j}} \notin S_{\pi_{j}}$, so $\operatorname{deg}\left(\pi_{j}\right) \leqslant n-j$. The only case in which it is possible is when $\pi_{j}=\delta_{j}$ and $\operatorname{deg}\left(\delta_{j}\right)=n-j$.

Finally, either all elements of $G$ are zeros of $h$ and $C T[f]=0$ or $\pi=\delta$ and $\operatorname{deg}\left(\delta_{i}\right)=$ $n-i$ for all $i$. If the latter is the case, obviously $T$ is transitive and $\pi=\delta=\sigma$.

The only thing left is to calculate the coefficient in the case of transitive $T$. We will omit the calculations here since they are given in a more general case in the next theorem.

The main result is the following theorem.

Theorem 4. Let $k,\left\{l_{i}\right\}_{i=1}^{k},\left\{m_{i}\right\}_{i=1}^{k},\left\{r_{i}\right\}_{i=1}^{k}$ be integers such that $1 \leqslant l_{1} \leqslant m_{1} \leqslant r_{1}<$ $l_{2} \leqslant \ldots \leqslant r_{k-1}<l_{k} \leqslant m_{k} \leqslant r_{k} \leqslant n$. Let

$$
\begin{aligned}
C_{i} & =\bigcup_{j=m_{i}+1}^{r_{i}}\left[l_{i}, j-2\right] \times\{j\}, B_{i} \subseteq C_{i}, \\
U_{i} & =\left(B_{i} \cup\left(\left[l_{i}, m_{i}-1\right] \times\left\{m_{i}\right\}\right) \cup \bigcup_{j=m_{i}}^{r_{i}-1}(j, j+1)\right), \\
U & =\bigcup_{i=1}^{k} U_{i} .
\end{aligned}
$$

Let $a_{1}, \ldots, a_{n}$ be positive integers, $a=a_{1}+\cdots+a_{n}$. Consider the Laurent polynomial

$$
f\left(x_{1}, \ldots, x_{n}, q\right)=\prod_{1 \leqslant i<j \leqslant n}\left(x_{i} / x_{j}\right)_{a_{i}}\left(q x_{j} / x_{i}\right)_{a_{j}-\chi((i, j) \in U)}
$$

over a field $\mathbb{F}$. Then

$$
C T[f]=\frac{(q)_{a}}{(q)_{a_{1}}(q)_{a_{2}} \cdots(q)_{a_{n}}} \times \prod_{i=1}^{k} \prod_{j=m_{i}}^{r_{i}} \frac{1-q^{a_{j}}}{1-q^{a_{i}+\cdots+a_{j}}} .
$$

Remark 5. The statement of the theorem is long and cumbersome, therefore we provide an illustration that can help to understand the idea behind the formal definitions.

Consider the correspondence between a Laurent polynomial

$$
f\left(x_{1}, \ldots, x_{n}, q\right)=\prod_{1 \leqslant i<j \leqslant n}\left(x_{i} / x_{j}\right)_{a_{i, j}}\left(q x_{j} / x_{i}\right)_{a_{j, i}}
$$

and a square matrix of non-negative integers with zeroes on the main diagonal $A=$ $\left\{a_{i, j}\right\}_{1 \leqslant i, j \leqslant n}$.

The polynomial from Theorem 2 corresponds to the matrix

$$
\left(\begin{array}{ccccc}
0 & a_{1} & a_{1} & \ldots & a_{1} \\
a_{2} & 0 & a_{2} & \ldots & a_{2} \\
a_{3} & a_{3} & 0 & \ldots & a_{3} \\
\ldots & \ldots & \ldots & \ldots & \ldots \\
a_{n} & a_{n} & \ldots & a_{n} & 0
\end{array}\right) .
$$


The polynomial from transitive part of Theorem 3 (for winner permutation $\sigma=i d$ ) corresponds to the matrix

$$
\left(\begin{array}{ccccc}
0 & a_{1} & a_{1} & \ldots & a_{1} \\
a_{2}-1 & 0 & a_{2} & \ldots & a_{2} \\
a_{3}-1 & a_{3}-1 & 0 & \ldots & a_{3} \\
\ldots & \ldots & \ldots & \ldots & \ldots \\
a_{n}-1 & a_{n}-1 & \ldots & a_{n}-1 & 0
\end{array}\right) .
$$

The polynomial from Theorem 4 in the case $n=9, k=1, l_{1}=2, m_{1}=5, r_{1}=8$, $B_{1}=\{(2,6),(4,6),(3,7),(4,7),(2,8),(4,8)\}$ corresponds to the matrix

$$
\left(\begin{array}{ccccccccc}
0 & a_{1} & a_{1} & a_{1} & a_{1} & a_{1} & a_{1} & a_{1} & a_{1} \\
a_{2} & 0 & a_{2} & a_{2} & a_{2} & a_{2} & a_{2} & a_{2} & a_{2} \\
a_{3} & a_{3} & 0 & a_{3} & a_{3} & a_{3} & a_{3} & a_{3} & a_{3} \\
a_{4} & a_{4} & a_{4} & 0 & a_{4} & a_{4} & a_{4} & a_{4} & a_{4} \\
a_{5} & a_{5}-1 & a_{5}-1 & a_{5}-1 & 0 & a_{5} & a_{5} & a_{5} & a_{5} \\
a_{6} & a_{6}-1 & a_{6} & a_{6}-1 & a_{6}-1 & 0 & a_{6} & a_{6} & a_{6} \\
a_{7} & a_{7} & a_{7}-1 & a_{7}-1 & a_{7} & a_{7}-1 & 0 & a_{7} & a_{7} \\
a_{8} & a_{8}-1 & a_{8} & a_{8}-1 & a_{8} & a_{8} & a_{8}-1 & 0 & a_{8} \\
a_{9} & a_{9} & a_{9} & a_{9} & a_{9} & a_{9} & a_{9} & a_{9} & 0
\end{array}\right) .
$$

As we can see, this matrix is the deformed version of the matrix from Theorem 2, with some coefficients decreased. Decreased coefficients are grouped into $k$ blocks. Each block consists of a segment of some row $\left(\left[l_{i}, m_{i}-1\right] \times\left\{m_{i}\right\}\right)$, a segment of the diagonal (the one under the main diagonal, $\left.\bigcup_{j=m_{i}}^{r_{i}-1}(j, j+1)\right)$ and an arbitrary subset of elements "under" them $\left(B_{i}\right.$ is an arbitrary subset of $\left.C_{i}\right)$.

Remark 6 . This theorem gives Theorem 2 when $k=0$. It also gives transitive part of Theorem 3 (for winner permutation $\sigma=i d$ ) when $k=1, l_{1}=m_{1}=1, r_{1}=n, B_{1}=C_{1}$.

Remark 7. This is a generalization of Theorem 2.5 from [2]. Specifically, it gives the said theorem when $B_{i}=C_{i}$ for all $i$.

Proof. $C T[f]$ equals to the coefficient of

$$
\prod_{i=1}^{n} x_{i}^{a-a_{i}-\sum_{j=1}^{n} \chi((i, j) \in U)}
$$

in the polynomial $h$, where

$$
h\left(x_{1}, \ldots, x_{n}, q\right)=\prod_{1 \leqslant i<j \leqslant n}\left(x_{i} / x_{j}\right)_{a_{i}}\left(q x_{j} / x_{i}\right)_{a_{j}-\chi((i, j) \in U)} \times x_{j}^{a_{i}} x_{i}^{a_{j}-\chi((i, j) \in U)} .
$$

We will use Theorem 1 again. Consider the grid

$$
G=\left\{\left(q^{y_{1}}, \ldots, q^{y_{n}}\right) \mid y_{i} \in R_{i}\right\},
$$


where

$$
\begin{gathered}
R_{i}=\left[0, a-a_{i}\right] \backslash S_{i}, \\
S_{i}=\left\{a-a_{i}-\sum_{v=r_{t}+1}^{n} a_{v}\right\} \cup\left\{a-a_{i}-\sum_{v=j}^{n} a_{v} \mid(i, j) \in B_{t}\right\},
\end{gathered}
$$

if there is a $t$ such that $i \in\left[l_{t}, r_{t}-1\right], S_{i}=\varnothing$ otherwise.

Denote $A \rtimes B=\{(i, j) \in A \times B \mid i<j\}$.

Consider

$$
N=[1, n] \rtimes[1, n], V_{i}=\left[l_{i}, r_{i}\right] \rtimes\left[m_{i}, r_{i}\right], V=\bigcup_{i=1}^{k} V_{i} .
$$

Note that $U_{i} \subseteq V_{i}$, and thus $U \subseteq V$. Also note that $C_{i} \backslash B_{i}=V_{i} \backslash U_{i}$.

We replace the linear factors of $h$

$$
\left(x_{i}-q^{a_{j}} x_{j}\right), \text { where }(i, j) \in V \backslash U
$$

by

$$
\left(x_{i}-q^{a_{j}} x_{j}-\left(q^{a-a_{i}-\sum_{v=j}^{n} a_{v}}-q^{a-\sum_{v=j}^{n} a_{v}}\right)\right),
$$

and call the modified polynomial $h^{\prime}$. The coefficient of $h$ we are interested in coincides with the corresponding coefficient of $h^{\prime}$ because it has the maximal sum of degrees of $x_{i}$, and the polynomials differ only by constants in linear factors.

Assume that $h^{\prime}$ does not vanish at $x=q^{y} \in G$. Let

$$
\chi_{1}(i, j)=\chi\left((i, j) \in V \backslash U \text { and } y_{i}=a-a_{i}-\sum_{v=j}^{n} a_{v} \text { and } y_{j}=a-\sum_{v=j}^{n} a_{v}\right) .
$$

For each $i<j$ either $y_{j}-y_{i} \geqslant a_{i}+\chi_{1}(i, j)$ or $y_{i}-y_{j} \geqslant a_{j}+\chi((i, j) \in N \backslash V)$ (otherwise one of the linear factors of $h^{\prime}$ is zero).

It follows that all $y_{i}$ are pairwise distinct. Let $\pi \in S_{n}$ be such a permutation that

$$
y_{\pi_{1}}<y_{\pi_{2}}<\cdots<y_{\pi_{n}} .
$$

Since $U \subseteq V$, we know that

$$
y_{\pi_{i+1}}-y_{\pi_{i}} \geqslant a_{\pi_{i}}+\chi\left(\left(\pi_{i+1}, \pi_{i}\right) \in N \backslash V\right)+\chi_{1}\left(\pi_{i}, \pi_{i+1}\right) .
$$

Adding up these inequalities and taking into account that $y_{\pi_{1}} \geqslant 0$, we get

$$
y_{\pi_{n}} \geqslant a-a_{\pi_{n}}+\sum_{i=1}^{n-1}\left(\chi\left(\left(\pi_{i+1}, \pi_{i}\right) \in N \backslash V\right)+\chi_{1}\left(\pi_{i}, \pi_{i+1}\right)\right) .
$$

But $y_{\pi_{n}} \leqslant a-a_{\pi_{n}}$, so $\left(\pi_{i+1}, \pi_{i}\right) \notin N \backslash V$ and $\chi_{1}\left(\pi_{i}, \pi_{i+1}\right)=0$ for all $i$.

Note that all intermediate inequalities have to become equalities, so

$$
y_{\pi_{i}}=a-\sum_{j=i}^{n} a_{\pi_{j}} .
$$


Let us denote the event $\pi_{i+1}<\pi_{i}$ as the descent. The descent is possible only when $\left(\pi_{i+1}, \pi_{i}\right) \in V$. From the definition of $V$ it follows that descents happen only if $\pi_{i}, \pi_{i+1} \in$ $\left[l_{t}, r_{t}\right]$ for some $t$. Then for each $t$ all elements of $\pi$ in the range $\left[l_{t}, r_{t}\right]$ should go in a row, all elements less than them should go before them, and all elements bigger should go after.

We will show that elements from $\left[l_{t}, r_{t}\right]$ go not just in a row, but in the ascending order. Therefore the only possible choice for $\pi$ is $i d$, and there is only one point on the grid at which $h^{\prime}$ does not vanish.

If $\pi_{r_{t}} \in\left[l_{t}, r_{t}-1\right]$, then

$$
y_{\pi_{r_{t}}}=a-a_{\pi_{r_{t}}}-\sum_{j=r_{t}+1}^{n} a_{\pi_{j}}=a-a_{\pi_{r_{t}}}-\sum_{j=r_{t}+1}^{n} a_{j},
$$

which contradicts the definition of $R_{\pi_{r_{t}}}$. So, $\pi_{r_{t}}=r_{t}$.

Consider $s \geqslant m_{t}$, and we have already showed that $\pi_{s+1}=s+1, \ldots, \pi_{r_{t}}=r_{t}$. Let us assume that $\pi_{s}<s$.

$\chi_{1}\left(\pi_{s}, \pi_{s+1}\right)=\chi_{1}\left(\pi_{s}, s+1\right)=0$. Additionally,

$$
y_{\pi_{s}}=a-a_{\pi_{s}}-\sum_{j=s+1}^{n} a_{j}, y_{s+1}=a-\sum_{j=s+1}^{n} a_{j}
$$

and $\left(\pi_{s}, s+1\right) \in V$, then from the definition of $\chi_{1}$ it follows that $\left(\pi_{s}, s+1\right) \in U . \pi_{s}<s$, so $\left(\pi_{s}, s+1\right) \in B_{t}$. But

$$
y_{\pi_{s}}=a-a_{\pi_{s}}-\sum_{j=s+1}^{n} a_{j}
$$

which contradicts the definition of $R_{\pi_{s}}$. So, $\pi_{s}=s$.

We proved that $\pi_{m_{t}}=m_{t}, \ldots, \pi_{r_{t}}=r_{t}$. By the definition of $V$, no descents are possible when $\pi_{i}, \pi_{i+1} \in\left[l_{t}, m_{t}-1\right]$, so all elements of $\left[l_{t}, m_{t}-1\right]$ also go in the ascending order.

So, $\pi=i d$, the only element of $G$ which is not a zero of $h^{\prime}$ is $x=q^{y}$, where $y=$ $\left(0, a_{1}, a_{1}+a_{2}, \ldots, a_{1}+\cdots+a_{n-1}\right)$.

Let us see what changes happen to the calculation of the coefficient compared to Theorem 2. For convenience denote $y_{n+1}=a$.

Fix $1 \leqslant t \leqslant k$.

Firstly, elements of $S_{i}\left(l_{t} \leqslant i<r_{t}\right)$ disappear from $R_{i}$, so the coefficient increases by a factor of

$$
\left(\prod_{i=l_{t}}^{r_{t}-1}\left(q^{y_{i}}-q^{a-a_{i}-\sum_{v=r_{t}+1}^{n} a_{v}}\right)\right) \times \prod_{(i, j) \in B_{t}}\left(q^{y_{i}}-q^{a-a_{i}-\sum_{v=j}^{n} a_{v}}\right) .
$$

Secondly, we add linear factor

$$
\left(x_{i}-q^{a_{j}} x_{j}-\left(q^{a-a_{i}-\sum_{v=j}^{n} a_{v}}-q^{a-\sum_{s=j}^{n} a_{v}}\right)\right)
$$


for all $(i, j) \in V_{t} \backslash U_{t}=C_{t} \backslash B_{t}$, so the coefficient increases by a factor of

$$
\prod_{(i, j) \in C_{t} \backslash B_{t}}\left(q^{y_{i}}-q^{y_{j+1}}-\left(q^{a-a_{i}-\sum_{v=j}^{n} a_{v}}-q^{a-\sum_{v=j}^{n} a_{v}}\right)\right)=\prod_{(i, j) \in C_{t} \backslash B_{t}}\left(q^{y_{i}}-q^{a-a_{i}-\sum_{v=j}^{n} a_{v}}\right) .
$$

Thirdly, we remove linear factor $\left(x_{i}-q^{a_{j}} x_{j}\right)$ for all $(i, j) \in V_{t}$, so the coefficient decreases by a factor of

$$
\prod_{(i, j) \in V_{t}}\left(q^{y_{i}}-q^{y_{j+1}}\right)
$$

In total, the coefficient increases by a factor of

$$
\begin{aligned}
& \prod_{i=l_{t}}^{r_{t}-1}\left(q^{y_{i}}-q^{a-a_{i}-\sum_{v=r_{t}+1}^{n} a_{v}}\right) \times \prod_{(i, j) \in C_{t}}\left(q^{y_{i}}-q^{a-a_{i}-\sum_{v=j}^{n} a_{v}}\right) /\left(\prod_{(i, j) \in V_{t}}\left(q^{y_{i}}-q^{y_{j+1}}\right)\right) \\
= & \prod_{j=m_{t}}^{r_{t}} \prod_{i=l_{t}}^{j-1}\left(q^{y_{i}}-q^{a-a_{i}-\sum_{v=j+1}^{n} a_{v}}\right) /\left(\prod_{j=m_{t}}^{r_{t}} \prod_{i=l_{t}}^{j-1}\left(q^{y_{i}}-q^{y_{j+1}}\right)\right) \\
= & \prod_{j=m_{t}}^{r_{t}} \prod_{i=l_{t}}^{j-1}\left(1-q^{a_{i+1}+\cdots+a_{j}}\right) /\left(\prod_{j=m_{t}}^{r_{t}} \prod_{i=l_{t}}^{j-1}\left(1-q^{a_{i}+\cdots+a_{j}}\right)\right) \\
= & \prod_{j=m_{t}}^{r_{t}} \prod_{i=l_{t}+1}^{j}\left(1-q^{a_{i}+\cdots+a_{j}}\right) /\left(\prod_{j=m_{t}}^{r_{t}} \prod_{i=l_{t}}^{j-1}\left(1-q^{a_{i}+\cdots+a_{j}}\right)\right) \\
= & \prod_{j=m_{t}}^{r_{t}} \frac{1-q^{a_{j}}}{1-q^{a_{l}+\cdots+a_{j}} .}
\end{aligned}
$$

All that remains is to multiply the results for $1 \leqslant t \leqslant k$.

\section{References}

[1] N. Alon. Combinatorial Nullstellensatz. Combin. Probab. Comput., 8:7-29, 1999.

[2] D. M. Bressoud and I. P. Goulden. Constant term identities extending the q-Dyson theorem. Trans. Amer. Math. Soc., 291:203-228, 1985.

[3] I. Cherednik. Double Affine Hecke Algebras and Macdonald's Conjectures. Ann. of Math., 141(1):191-216, 1995.

[4] F. J. Dyson. Statistical theory of energy levels of complex systems. I. J. Math. Phys., 3:140-156, 1962.

[5] S. B. Ekhad and D. Zeilberger. How to Extend Károlyi and Nagy's BRILLIANT Proof of the Zeilberger-Bressoud q-Dyson Theorem in order to Evaluate ANY Coefficient of the q-Dyson Product. Personal Journal of Shalosh B. Ekhad and Doron Zeilberger., see also arXiv:1308.2983, 2013. 
[6] P. J. Forrester and S. O. Warnaar. The importance of the Selberg integral. Bull. Amer. Math. Soc. (N.S.), 45:489-534, 2008.

[7] I. J. Good. Short proof of a conjecture by Dyson. J. Math. Phys., 11:1884, 1970.

[8] K. G. Jacobi. Theoremata nova algebraica circa systema duarum aequationum inter duas variabiles propositarum. J. Reine Angew. Math., 14:281-288, 1835.

[9] E. Kunz and M. Kreuzer. Traces in strict Frobenius algebras and strict complete intersections. J. reine angew. Math., 381:181-204, 1987.

[10] G. Károlyi, A. Lascoux, and S. O. Warnaar. Constant term identities and Poincaré polynomials. Trans. Amer. Math. Soc., 367:6809-6836, 2015.

[11] G. Károlyi and Z. L. Nagy. A simple proof of the Zeilberger-Bressoud q-Dyson theorem. Proc. Amer. Math. Soc., 142:3007-3011, 2014.

[12] G. Károlyi, Z. L. Nagy, F. V. Petrov, V. Volkov. A new approach to constant term identities and Selberg-type integrals. Adv. Math., 277:252-282, 2015.

[13] R. N. Karasev, F. V. Petrov. Partitions of nonzero elements of a finite field into pairs. Israel J. Math., 192(1):143-156, 2012.

[14] M. Lasoń. A generalization of Combinatorial Nullstellensatz. Electron. J. Combin., 17(1), \#N32, 6 pages, 2010.

[15] U. Schauz. Algebraically solvable problems: describing polynomials as equivalent to explicit solutions. Electron. J. Combin., 15, \#R10, 35 pages, 2008.

[16] K. G. Wilson. Proof of a conjecture by Dyson. J. Math. Phys., 3:1040-1043, 1962.

[17] D. Zeilberger and D. M. Bressoud. A proof of Andrews' q-Dyson conjecture. Discrete Math., 54:201-224, 1985. 